\section{Schlüsselerlebnis beim Kopfball}

— Im Juni 2008 wurde ein 15-jähriger Junge vom Sportplatz in die Nothilfe gebracht. In seiner linken Augenhöhle steckte ein Schlüssel (Abb. A, B).

Der Junge hatte Fußball gespielt. Dabei hatte er an einer Kette einen Schlüssel um den Hals hängen. Bei einem Kopfballversuch sprang er hoch, der Schlüssel wurde an der Kette hängend nach oben geschleudert, geriet durch einen unglücklichen Zufall genau zwischen den heranfliegenden Ball und den Kopf des Jungen und bohrte sich auf diese Weise in die linke Augenhöhle.

Das Computertomogramm zeigte, dass der Schlüssel zwar tief in der Augenhöhle steckte, jedoch glücklicherweise den Augapfel nicht verletzt hatte (Abb. C). Der Schlüssel wurde operativ entfernt und der Junge prophylaktisch mit Antibiotika behandelt. Es grenzt an ein Wunder, dass der Visus des Jungen postoperativ nicht beeinträchtigt war.
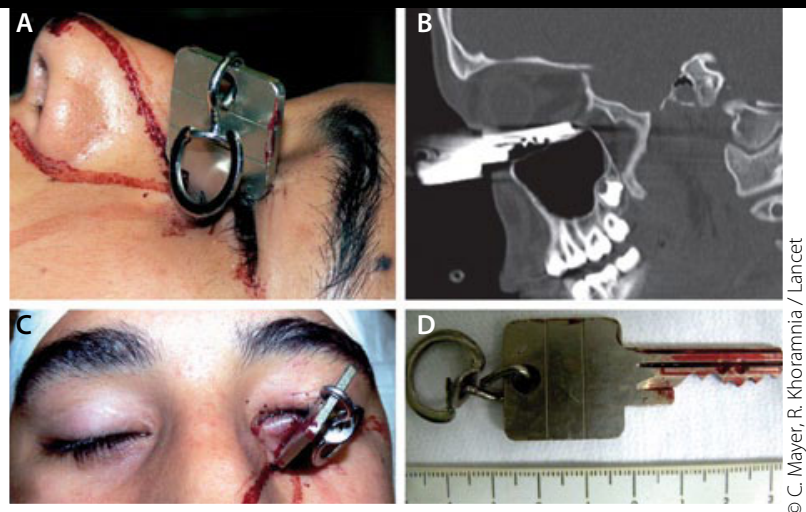

Wie Bild B zeigt, ist der Bulbus nicht verletzt.

diesem Fall nicht als Schmuck, sondern zur Aufbewahrung des Schlüssels, doch hätte zum Beispiel ein kleines Kreuz durchaus einen ähnlichen Effekt haben können. Auch Ohrringe oder Armbänder sind ebenso wie Brillen beim Spiel nicht erlaubt. In einem makaberen Wortspiel nennen die Autoren die Verletzung einen „eye-catcher". Das Auge des Jungen hat der Schlüssel zwar nicht "gefangen", dafür aber vermutlich die Aufmerksamkeit des gesamten Klinikpersonals auf sich gezogen.

H. S. FÜESSL

\section{- C. Mayer, R. Khoramnia}

(Eye Clinic Klinikum rechts der Isar der technischen Universität München, Ismaninger Straße 22,81675 Munich): Nearly an eye-catcher. Lancet 377 (2011) 9777, 1599

\title{
Patienten mit resistenter Hypertonie: älter, dicker und komorbider
}

\begin{abstract}
Patienten mit resistenter Hypertonie unterscheiden sich in einigen klinischen Merkmalen von Hypertonikern, deren Blutdruck medikamentös unter Kontrolle zu bringen ist.
\end{abstract}

_ Für die Studie wurden Daten von 15968 Probanden aus dem National Health and Nutrition Examination Survey (NHANES) von 2003 bis 2008 ausgewertet. Eine Hypertonie wurde als resistent bezeichnet, wenn der Blutdruck bei Therapie mit drei oder mehr Antihypertensiva entweder systolisch $\geq 140 \mathrm{mmHg}$ oder diastolisch $\geq 90 \mathrm{mmHg}$ lag oder unabhängig von den Blutdruckwerten, wenn vier oder mehr Antihypertensiva notwendig waren.

In dieser Erhebung zeigten sich die resistenten Hypertoniker signifikant älter und adipöser. Ferner hatten sie signifikant häufiger eine Albuminurie, eine

verminderte Nierenfunktion und (entsprechend den anamnestischen Daten) häufiger eine KHK, Herzinsuffizienz, Diabetes mellitus und Schlaganfall.

\section{Kommentar}

Für die praktische Hypertoniebehandlung wichtige Befunde bzw. Schlussfolgerungen sind:

- Die resistente Hypertonie tritt etwa bei 10\% der Hypertoniker auf und ist infolge der hohen Gesamtzahl der Hypertoniker ein signifikantes Problem.

- Endorganschäden an Herz und Gehirn wie auch an den Nieren sind bei resistenter Hypertonie häufiger. Es bleibt offen, was hier Henne oder Ei ist: Einerseits könnte die signifikant stärkere Nierenschädigung eine Folge der resistenten Hypertonie sein, andererseits mag auch durch die vermin-
- S. D. Persell et al.

Prevalence of Resistant Hypertension in the United States, 2003-2008. Hypertension 57 (2011) 1076-1080 